\title{
Estimation of the number of hypotheses in a concept learning task
}

\author{
HERBERT WELLS AND JAMES FOREST ${ }^{2}$ \\ UNIVERSITY OF WASHINGTON
}

Bayesian probability-revision formulae comprise a normative model for concept learning tasks. In an initial study suggested by this consideration, $48 \mathrm{Ss}$ estimated the number of tenable hypotheses remaining on each of 13 trials. On a separate task the same Ss gave one hypothesis on each trial, together with a confidence judgment. Median scores indicated considerable accuracy on both tasks following the first three trials.

Information theory has been considered the normative model for concept learning (CL) tasks since Hovland's (1952) theoretical paper. The model specifies the actual trial by trial reduction in tenable hypotheses concerning the concept, and hence the trial number on which a maximally efficient information processor could attain the concept.

Several authors (Hunt, 1962; Suppes, 1966; Tikhomirov, 1961) have pointed out that Bayesian probabilityrevision formulae can also be considered normative models for CL. The probability of each hypothesis (H) given the most recent datum (the instance and its class membership) is specified by the formula:

$$
\mathbf{P}(\mathrm{H} \mid \mathrm{D})=\frac{\mathbf{P}(\mathrm{D} \mid \mathrm{H}) \cdot \mathbf{P}(\mathrm{H})}{\mathbf{P}(\mathrm{D})}
$$

where $P(H \mid D)$ is the conditional probability of the hypothesis given the current instance; $P(D \mid H)$ is the probability of the instance's class assignment if the hypothesis were true (either 1 or 0 except in the case of probabilistic $C L) ; P(H)$ is the probability of the hypothesis prior to the current instance; and $P(D)$ is the a prfori probabllity of the instance being a member of the class ( .5 in the case of two category CL). In the simplest case, $P(H \mid D)$ will be either 0 or $1 / N_{h}$, where $\mathrm{N}_{h}$ is the number of hypotheses which remain tenable. As Hunt points out (1962, pp. $35 \mathrm{ff}), S^{\prime} \mathrm{s}$ initial blas toward certain hypotheses can be reflected in continuing inequalities among the nonzero $P(H \mid D)$.

In the simplest case-that of deterministic rather than probabllistic CL, and where there is no evidence of systematic variations in the prior probabilities of the several hypotheses-the Bayesian and the information models are equivalent normative models. Since the Bayesian model is clearly more cumbersome, it is reasonable to ask whether it constitutes anything more than an intellectual curiosity for CL research. But the Bayesian approach in general suggests some interesting empirical problems which may make its consideration worthwhile. In particular, the notion of intuitive estimation of various parameters has been explored in several other experimental realms (Peterson \& Beach, 1967).
The typical CL experiment employs measures such as trials or errors to a criterion, or the statement of a single hypothesis by $S$ on a given trial. Ss are usually less efficient than the normative information model by such measures.

But could Ss also attach a probability value to their current hypothesis, a kind of intuitive $P(H \mid D)$ ? How would they compare with the model in estimating $N_{h}$, the reciprocal of $P(H \mid D)$ ? Do they appreciate the amount of information transmitted by successive instances, regardless of their inability to use all of it?

Several additional questions could be raised about performance on such a task. What is the relation between estimates of $\mathrm{N}_{h}$ and performance on a standard CL task involving the same instances? Is S's confidence in his current hypothesis in a CL task related to his estimate of $\mathrm{N}_{\mathrm{h}}$ ? What influence does previous experience in a CL task have on accuracy of estimating $\mathrm{N}_{\mathrm{h}}$ ? This experiment was intended to explore some of the implications and potentials of such estimation measures in $\mathrm{CL}$.

Method

The stimulus array was composed of geometrical figures with six binary dimensions: color, shape, size, number of figures, internal shading, and number of borders. These were presented by a $35 \mathrm{~mm}$ projector to groups of two to four Ss. The stimulus for each trial remained on until all Ss had responded (10-15 $\mathrm{sec})$. Positive and negative instances were indicated by a plus or minus in the corner of the slide. Two different conjunctive concepts were used, differing in relevant dimensions. In every case a problem consisted of 13 stimuli, both positive and negative instances. The concept was completely specified after nine instances.

Ss were first given an example problem with one relevant dimension, and were shown that hypotheses are eliminated by successive slides until eventually only one (the correct answer) remains. On the actual problems Ss were told that the concept was a two valued conjunction and that there were 60 hypotheses at the outset.

In the estimation task $S$ indicated his guess about $\mathrm{N}_{\mathrm{h}}$ after each slide by marking a scale numbered 1-60. One scale appeared on each page of a 13 page booklet. In the $C L$ task, $S$ was encouraged to guess about the concept, and to indicate his guess on the appropriate page of a booklet. He also indicated his confidence in that hypothesis by marking a scale numbered 0-100.

Forty-eight students from introductory psychology classes served as Ss. Each $\mathrm{S}$ performed both the 
estimation and the CL task, with a different concept on the two tasks. Order of tasks and of concepts, as well as assignment of concepts to tasks, was counterbalanced. Group A received the CL task followed by the estimation task, and for Group B this order was reversed.

\section{Resulis}

Figure 1 presents the median $\mathrm{N}_{\mathrm{h}}$ estimates of the two order groups, and the objective $\mathrm{N}_{h}$, for each trial. A median-split chi square analysis on each trial separately indicates that $\mathrm{Ss}$ in Group $\mathrm{B}$ gave lower estimates on Trials $1-6$ and 8 (chi square values $\geq 4.083$, $\mathrm{p}<.05)$. Ss' estimates most clearly exceeded the model on the first trial, when only five of 48 Ss gave estimates as small as the actual $\mathrm{N}_{\mathrm{h}}$.

Figure 2 shows performance on the CL task in terms of the cumulative percent of Ss who offer the correct hypothesis on each trial. For comparison, the optimum probability of solution is given by the reciprocal of the true $\mathrm{N}_{\mathrm{h}}$ on each trial. The median confidence judgments are also plotted on the same scale. Confidence data are based on 36 Ss (nine in each concept/order subgroup) who began giving confidence judgments by Trial 4. These 36 Ss were indistinguishable from the full group in terms of accuracy of performance on the

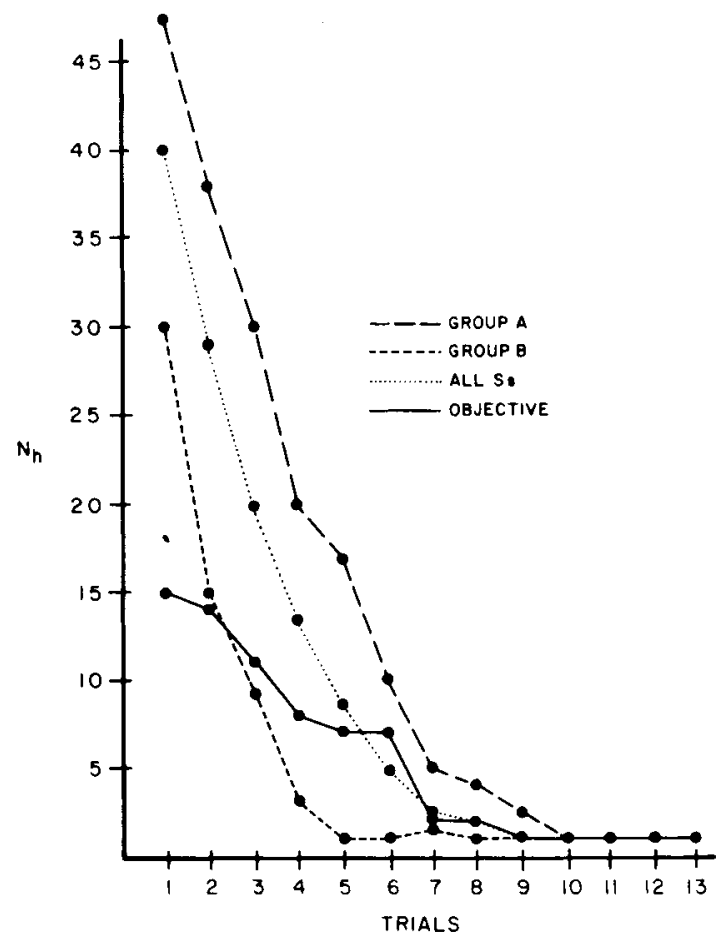

Fig. 1. Number of remaining hypotheses $\left(\mathrm{N}_{\mathrm{h}}\right)$ : median estimates by $\mathrm{Ss}$ compared with objective $N_{h}$.

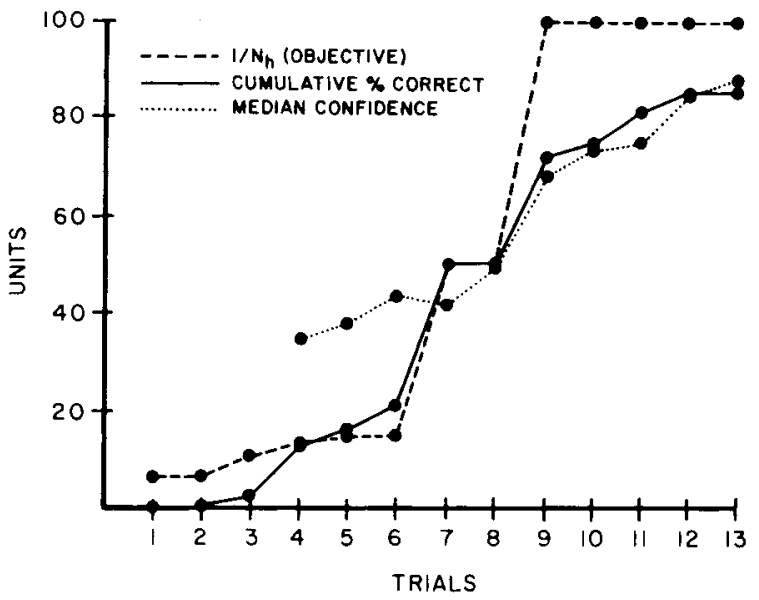

Fig. 2. 1/N $\mathrm{N}_{\mathrm{h}}$ (objective); Cumulative $\%$ correct; and median confidence scores, plotted on the same scale.

CL task, and estimation scores on the other task. Discussion

On the first few trials, when the true $\mathrm{N}_{h}$ is rapidly reduced, Ss tend to be conservative in their estimates of reduction. But on the whole they are more accurate than one would have expected, since both the model and the median $\mathrm{S}$ reach $\mathrm{N}_{\mathrm{h}}=1$ on the same trial. The effect of task order suggests that experience with a CL task makes Ss more rather than less conservative estimators. The fit between the model and the data on the CL task is quite close, up to the point at which full information is imparted.

In this initial study the effects of trial number, amount of information, and positive vs negative instances were confounded, but could be separated in further experiments.

\section{References}

HOVLAND, C. I. A "communication analysis" of concept learning. Psychol. Rev., 1952, 59, 461-472.

HUNT, E. B. Concept learning. New York: Wiley, 1962.

PETERSON, C. R., \& BEACH, L. R. Man as an intuitive statistician. Psychol. Bull, 1967, 68, 29-46.

SUPPES, P. Concept formation and Bayesian decisions. In K. J. J. Hintikka \& P. Suppes (Eds.), A spects of inductive logic. Amsterdam: North Holland Publishing Company, 1966.

TIKHOMIROV, O. K. The solution of thinking problems as a probability process. Voprosy Psikhologii, 1961, No. 5. (Trans. in: Soviet psychology and psychiatry, 1962, 1, 21-30.)

Notes

1. Supported in part by USPHS grant No. MH 12309-01, and Air Force Office of Scientific Research grant No. 1311-67.

2. The second author held a University of Washington Graduate School Research Assistantship. We thank Lee R. Beach for discussions and comments at several stages of the study. 